\title{
Show off the efficiency of dai-liao method in merging technology for monotonous non-linear problems
}

\author{
Rana Z. Al-Kawaz, Abbas Y. Al-Bayati \\ Department of Mathematics, College of Basic Education, University of Telafer, Mosul, Iraq
}

\begin{tabular}{ll}
\hline \hline Article Info & ABSTRACT \\
\cline { 1 - 2 } $\begin{array}{l}\text { Article history: } \\
\text { Received May 16, } 2020\end{array}$ & $\begin{array}{l}\text { In this article, we give a new modification for the Dai-Liao method to solve } \\
\text { monotonous nonlinear problems. In our modification, we relied on two } \\
\text { important procedures, one of them was the projection method and the second } \\
\text { Accepted Aug 4, 2020 }\end{array}$ \\
$\begin{array}{l}\text { was the method of damping the quasi-Newton condition. The new approach } \\
\text { of derivation yields two new parameters for the conjugated gradient direction } \\
\text { which, through some conditions, we have demonstrated the sufficient descent } \\
\text { property for them. Under some necessary conditions, the new approach } \\
\text { achieved global convergence property. Numerical results show how efficient } \\
\text { the new approach is when compared with basic similar classic methods. }\end{array}$ \\
Dai-Liao method
\end{tabular}

This is an open access article under the CC BY-SA license.

Damping technology

Global convergence

Projection method

Quasi-Newton condition \begin{abstract}
monotonous nonlinear problems. In our modification, we relied on two important procedures, one of them was the projection method and the second which, through some conditions, we have demonstrated the sufficient descent achieved global convergence property. Numerical results show how efficient
\end{abstract}

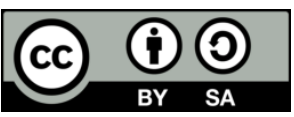

\section{Corresponding Author:}

Rana Z. Al-Kawaz

Department of Mathematics

University of Telafer

College of Basic Education, Mosul, Iraq

Email: rana.alkawaz@yahoo.com

\section{INTRODUCTION}

The issue in this article is the assumption of finding the vector value $\mathrm{x} \in \mathbb{R}^{n}$, i.e. as in:

$$
x \in \Omega, \quad F(x)=0
$$

When $F: \Omega \subset \mathbb{R}^{n} \rightarrow \mathbb{R}^{n}$ is continuous and monotonous and satisfy $(F(x)-F(y))^{T}(x-y) \geq 0$. Methods for solving this type of problem vary when they are not restricted to Newton's method and quasi-Newton methods, and they are preferred due to the convergence of their local lines to the second and local levels. When dealing with largescale nonlinear equations, the so-called Conjugate Gradient (CG) method of all kinds is effective [1-8]. Applications and innovations continue around these technologies to this day [9-11]. The monotonic equations arose in several different practical situations for example see [12]. The most important advantage of CG-methods is that the direction of the search does not require the calculation of the Jacobin matrix which leads to low math requirements on each iteration. Likewise, when these methods overlap with the projection technique proposed by Solodov and Svaiter [13] to solve large-scale nonlinear equations and constrained nonlinear equations that some researchers have expanded as in [14-19]. Recently, many researchers have presented articles on how to find the solution to both constrained and unconstrained monotones (1) and give them a lot of attention [20-27]. Include the idea of projection that needs to be accelerated using a monotonous case $\mathrm{F}$ by monotony $\mathrm{F}$ and letting $z_{k}=x_{k}+\alpha_{k} d_{k}$, the hyperplane:

$$
\mathrm{H}=\left\{x \in \mathbb{R}^{n} \mid F\left(z_{k}\right)^{T}\left(x-z_{k}\right)=0\right\} .
$$


Separates strictly $x_{k}$ from the solution set of (2). Through [13] where the next iteration $x_{k+1}$ to be the projection of $x_{k}$ onto the hyperplane $H_{k}$. So, $x_{k+1}$ can be evaluated as:

$$
\begin{gathered}
x_{k+1}=P_{\Omega}\left[x_{k}-\xi_{k} F\left(z_{k}\right)\right]=x_{k}-\frac{F\left(z_{k}\right)^{T}\left(x-z_{k}\right) F\left(z_{k}\right)}{\left\|F\left(z_{k}\right)\right\|^{2}} \\
\xi_{k}=\frac{F\left(z_{k}\right)^{T}\left(x_{k}-z_{k}\right)}{\left\|F\left(z_{k}\right)\right\|^{2}}
\end{gathered}
$$

This paper is organized as follows: In Section 2, we describe the proposed new procedure. Section 3 derived the penalty parameters. Global convergence has been demonstrated in Section 4. For Section 5 we list numerical experiments in it.

\section{OPTIMAL DAMPED DAI-LIAO}

The researchers gave Dai and Liao [28] a parameter worthy of updating to this time because of its ability to reach global convergence through the properties of parameter $t$, which could set us some failures resulting from the deviation of the search direction of its path to reach the smallest point of the function, such that:

$$
\beta_{k}^{D L}=\frac{g_{k+1}^{T}\left(y_{k}-t s_{k}\right)}{d_{k}^{T} y_{k}}
$$

Later Liu and Li modified (3) by using the projection technique in their formula [29]. The researcher Fatemi [30] presented a precise method in deriving the conjugate gradient parameter $\beta_{k}$ by setting conditions on it (the condition of orthogonality and conjugation) and through the penalty function the results of the derivation were largely appropriate in developing a formula for the Dai-Liao parameter and the positive value of $\mathrm{t}$. In this section, we present an improved method for deriving a parameter $\beta_{k}$. That is:

$$
q(d)=f_{k+1}+g_{k+1}^{T} d+\frac{1}{2} d^{T} B_{k+1} d
$$
see that:

And take $\nabla q\left(\alpha_{k+1} d_{k+1}\right)$, the gradient of the model in $x_{k+2}$, as an estimation of $g_{k+2}$. It is easy to

$$
\nabla q\left(\alpha_{k+1} d_{k+1}\right)=g_{k+1}+\alpha_{k+1} B_{k+1} d
$$

Unfortunately, $\alpha_{k+1}$ in (4) is not available in the current iteration, because $d_{k+1}$ is unknown. Thus, we modified (4), and set

$$
g_{k+2}=g_{k+1}+t B_{k+1} d
$$

Where $\mathrm{t}>0$ is suitable approximation of $\alpha_{k+1}$. If the search direction of CG-method such that

$$
d_{k+1}=-g_{k+1}+\beta_{k} d_{k}
$$

An efficient nonlinear CG-method, we introduce the following optimization problem based on the penalty function:

$$
\min _{\beta_{k}}\left[g_{k+1}^{T} d_{k+1}+P \sum_{i=0}^{m}\left[\left(g_{k+2}^{T} s_{k-i}\right)^{2}+\left(d_{k+1}^{T} y_{k-i}\right)^{2}\right]\right]
$$

Now, substituting (5) and (6) in (7), and use the projection technique we obtain:

$$
\begin{aligned}
\min _{\beta_{k}}\left[-\left\|F_{k+1}\right\|^{2}+\beta_{k}\right. & g_{k+1}^{T} d_{k} \\
& +P \sum_{i=0}^{m}\left[\left(F_{k+1}^{T} s_{k-i}\right)^{2}+2 t F_{k+1}^{T} s_{k-i} d_{k+1}{ }^{T} B_{k+1} S_{k-i}+t^{2}\left(d_{k+1}{ }^{T} B_{k+1} S_{k-i}\right)^{2}+\left(F_{k+1}^{T} y_{k-i}\right)^{2}-2 \beta_{k} F_{k+1}^{T} y_{k-i} d_{k}^{T} y_{k-i}\right. \\
& \left.\left.+\left(\beta_{k} d_{k}^{T} y_{k-i}\right)^{2}\right]\right]
\end{aligned}
$$


After some algebraic abbreviations, we get the following formula

$$
\begin{aligned}
\beta_{k}=\frac{1}{\varphi} & {\left[-F_{k+1}^{T} d_{k}+2 P \sum_{i=0}^{m} F_{k+1}^{T} y_{k-i} d_{k}^{T} y_{k-i}+2 t^{2} P \sum_{i=0}^{m} F_{k+1}^{T} B_{k+1} s_{k-i} d_{k}^{T} B_{k+1} s_{k-i}\right.} \\
& \left.\quad-2 t P \sum_{i=0}^{m} F_{k+1}^{T} S_{k-i} d_{k}^{T} B_{k+1} s_{k-i}\right]
\end{aligned}
$$

Where $\varphi=2 t^{2} P \sum_{i=0}^{m}\left(d_{k}^{T} B_{k+1} s_{k-i}\right)^{2}+2 P \sum_{i=0}^{m}\left(d_{k}^{T} y_{k-i}\right)^{2}$

To get a new parameter we consider the following assumption as the hessian approximation $B_{k+1}$ satisfies the extended damped quasi-Newton equation and with the incorporation of the use of projection technology we get:

$$
B_{k+1} s_{k-i}=\frac{1}{\xi_{k}}\left(\tau_{k} y_{k-i}+\left(1-\tau_{k}\right) B_{k} s_{k-i}\right)=\frac{1}{\xi_{k}} y_{k-i}^{D}=\bar{y}_{k-i}^{D}
$$

Where

$$
\tau_{k}= \begin{cases}1 & \text { if } s_{k-i}^{T} y_{k-i} \geq s_{k-i}^{T} B_{k} s_{k-i} \\ \frac{\eta s_{k-i}^{T} B_{k} s_{k-i}}{s_{k-i}^{T} B_{k} s_{k-i}-s_{k-i}^{T} y_{k-i}} & \text { if } s_{k-i}^{T} y_{k-i}<s_{k-i}^{T} B_{k} s_{k-i}\end{cases}
$$

And $\xi_{k}$ is the projection step. We get

$$
\begin{aligned}
\beta_{k}^{\text {new }}=\frac{-F_{k+1}^{T} d_{k}}{2 P \sum_{i=0}^{m}\left(t^{2}\left(d_{k}^{T} \bar{y}_{k-i}^{D}\right)^{2}+\left(d_{k}^{T} y_{k-i}\right)^{2}\right)}+\frac{\sum_{i=0}^{m} F_{k+1}^{T} y_{k-i} d_{k}^{T} y_{k-i}}{\sum_{i=0}^{m}\left(t^{2}\left(d_{k}^{T} \bar{y}_{k-i}^{D}\right)^{2}+\left(d_{k}^{T} y_{k-i}\right)^{2}\right)} \\
+\frac{t^{2} \sum_{i=0}^{m} F_{k+1}^{T} \bar{y}_{k-i}^{D} d_{k}^{T} \bar{y}_{k-i}^{D}}{\sum_{i=0}^{m}\left(t^{2}\left(d_{k}^{T} \bar{y}_{k-i}^{D}\right)^{2}+\left(d_{k}^{T} y_{k-i}\right)^{2}\right)}-\frac{t \sum_{i=0}^{m} F_{k+1}^{T} s_{k-i} d_{k}^{T} \bar{y}_{k-i}^{D}}{\sum_{i=0}^{m}\left(t^{2}\left(d_{k}^{T} \bar{y}_{k-i}^{D}\right)^{2}+\left(d_{k}^{T} y_{k-i}\right)^{2}\right)}
\end{aligned}
$$

So, there are two possible scenarios for this parameter such that:

Case I: if $s_{k-i}^{T} y_{k-i} \geq s_{k-i}^{T} B_{k} s_{k-i}$ then $\tau_{k}=1$ and $\bar{y}_{k-i}^{D}=\frac{y_{k-i}}{\xi_{k}}$

$$
\begin{aligned}
\beta_{k}^{\text {new } 1}= & \frac{-F_{k+1}^{T} d_{k}}{2 P \sum_{i=0}^{m}\left(\frac{t^{2}}{\xi_{k}^{2}}\left(d_{k}^{T} y_{k-i}\right)^{2}+\left(d_{k}^{T} y_{k-i}\right)^{2}\right)}+\frac{\sum_{i=0}^{m} F_{k+1}^{T} y_{k-i} d_{k}^{T} y_{k-i}}{\sum_{i=0}^{m}\left(\frac{t^{2}}{\xi_{k}^{2}}\left(d_{k}^{T} y_{k-i}\right)^{2}+\left(d_{k}^{T} y_{k-i}\right)^{2}\right)} \\
& +\frac{\frac{t^{2}}{\xi_{k}^{2}} \sum_{i=0}^{m} F_{k+1}^{T} y_{k-i} d_{k}^{T} y_{k-i}}{\sum_{i=0}^{m}\left(\frac{t^{2}}{\xi_{k}^{2}}\left(d_{k}^{T} y_{k-i}\right)^{2}+\left(d_{k}^{T} y_{k-i}\right)^{2}\right)}-\frac{\frac{t}{\xi_{k}} \sum_{i=0}^{m} F_{k+1}^{T} s_{k-i} d_{k}^{T} y_{k-i}}{\sum_{i=0}^{m}\left(\frac{t^{2}}{\xi_{k}^{2}}\left(d_{k}^{T} y_{k-i}\right)^{2}+\left(d_{k}^{T} y_{k-i}\right)^{2}\right)}
\end{aligned}
$$

If $s_{k}=\xi_{k} d_{k}$

$$
\beta_{k}^{\text {new } 1}=\frac{\sum_{i=0}^{m} F_{k+1}^{T} y_{k-i} d_{k}^{T} y_{k-i}}{\sum_{i=0}^{m}\left(d_{k}^{T} y_{k-i}\right)^{2}}-\frac{\xi_{k} t}{\left(t^{2}+1\right)} \frac{\sum_{i=0}^{m} F_{k+1}^{T} s_{k-i} d_{k}^{T} y_{k-i}}{\sum_{i=0}^{m}\left(d_{k}^{T} y_{k-i}\right)^{2}}-\frac{\xi_{k} F_{k+1}^{T} s_{k}}{2 P_{1}\left(t^{2}+1\right) \sum_{i=0}^{m}\left(d_{k}^{T} y_{k-i}\right)^{2}}
$$

It is interesting to investigate the method when $P_{1}$ approaches infinity, because by making this coefficient larger, we penalize the conjugacy condition and the orthogonality property violations more severely, thereby forcing the minimizer of (7) closer to that of linear conjugate gradient method. We obtain

$$
\beta_{k}^{\text {new } 1}=\frac{\sum_{i=0}^{m} F_{k+1}^{T} y_{k-i} d_{k}^{T} y_{k-i}}{\sum_{i=0}^{m}\left(d_{k}^{T} y_{k-i}\right)^{2}}-\frac{\xi_{k} t}{\left(t^{2}+1\right)} \frac{\sum_{i=0}^{m} F_{k+1}^{T} s_{k-i} d_{k}^{T} y_{k-i}}{\sum_{i=0}^{m}\left(d_{k}^{T} y_{k-i}\right)^{2}}
$$

We notice from the previous equation that it belongs to the parameter class for Dai-Liao and that's exactly when setting $\mathrm{m}=0$ we have 


$$
\begin{aligned}
& \beta_{k}^{\text {new1 }}=\frac{F_{k+1}^{T} y_{k}}{d_{k}^{T} y_{k}}-\frac{\xi_{k} t}{\left(t^{2}+1\right)} \frac{F_{k+1}^{T} s_{k}}{d_{k}^{T} y_{k}}-\frac{\xi_{k} F_{k+1}^{T} s_{k}}{2 P_{1}\left(t^{2}+1\right) d_{k}^{T} y_{k}} \\
& \beta_{k}^{\text {new } 1}=\frac{g_{k+1}^{T} y_{k}}{d_{k}^{T} y_{k}}-\frac{\xi_{k} t}{\left(t^{2}+1\right)} \frac{g_{k+1}^{T} s_{k}}{d_{k}^{T} y_{k}}
\end{aligned}
$$

When compared to the parameter Dai-Liao, we notice that the value is

$$
v=\frac{\xi_{k} t}{\left(t^{2}+1\right)}, \quad v \in\left(0, \frac{1}{2}\right]
$$

Case II: if $s_{k-i}^{T} y_{k-i}<s_{k-i}^{T} B_{k} s_{k-i}$ then $\bar{y}_{k-i}^{D}=\frac{y_{k-i}^{D}}{\xi_{k}}$ from equation (9) and $s_{k}=\xi_{k} d_{k}$ by projection technique, $\mathrm{m}=0$ to convert the $\beta_{k}^{\text {new }}$ form to

$$
\beta_{k}^{\text {new } 2}=\left[\frac{\frac{t^{2}}{\xi_{k}^{2}} F_{k+1}^{T} y_{k}^{D} d_{k}^{T} y_{k}^{D}}{\left(\frac{t^{2}}{\xi_{k}^{2}}\left(d_{k}^{T} y_{k}^{D}\right)^{2}+\left(d_{k}^{T} y_{k}\right)^{2}\right)}-\frac{\frac{t}{\xi_{k}} F_{k+1}^{T} s_{k} d_{k}^{T} y_{k}^{D}}{\left(\frac{t^{2}}{\xi_{k}^{2}}\left(d_{k}^{T} y_{k}^{D}\right)^{2}+\left(d_{k}^{T} y_{k}\right)^{2}\right)}\right]+\left[\frac{F_{k+1}^{T} y_{k} d_{k}^{T} y_{k}}{\left(\frac{t^{2}}{\xi_{k}^{2}}\left(d_{k}^{T} y_{k}^{D}\right)^{2}+\left(d_{k}^{T} y_{k}\right)^{2}\right)}-\frac{F_{k+1}^{T} s_{k}}{2 P_{2} \xi_{k}\left(\frac{t^{2}}{\xi_{k}^{2}}\left(d_{k}^{T} y_{k}^{D}\right)^{2}+\left(d_{k}^{T} y_{k}\right)^{2}\right)}\right] \text { (15) }
$$

If we substituting equation (9) in (15) and using algebraic simplifications, we obtain the formula:

$$
\beta_{k}^{\text {new } 2}=\frac{1}{\varphi^{d}}\left(\left[t_{1} F_{k+1}^{T} y_{k}-t_{2} F_{k+1}^{T} s_{k}\right]+\frac{d_{k}^{T} s_{k}}{d_{k}^{T} y_{k}}\left[t_{3} F_{k+1}^{T} y_{k}-t_{4} F_{k+1}^{T} s_{k}\right]-\frac{\xi_{k}}{2 P_{2} d_{k}^{T} y_{k}} F_{k+1}^{T} s_{k}\right)
$$

$$
\begin{aligned}
\text { i.e. } \varphi^{d} & =\left(t^{2}\left(d_{k}^{T} y_{k}^{D}\right)^{2}+\xi_{k}^{2}\left(d_{k}^{T} y_{k}\right)^{2}\right) \\
t_{1} & =t^{2} \tau_{k}^{2}+\xi_{k}^{2} \quad \text { and } t_{2}=t \xi_{k} \tau_{k}-t^{2} \tau_{k}\left(1-\tau_{k}\right) \\
t_{3} & =t^{2} \tau_{k}\left(1-\tau_{k}\right) \text { and } t_{4}=t \xi_{k}\left(1-\tau_{k}\right)-t^{2}\left(1-\tau_{k}\right)^{2}
\end{aligned}
$$

As we talked about $\left(P_{1}\right)$ then $\left(P_{2}\right)$ when you come close to infinity, then we use the parameter omitted from this limit:

$$
\beta_{k}^{\text {new } 2}=\frac{1}{\varphi^{d}}\left(\left[t_{1} F_{k+1}^{T} y_{k}-t_{2} F_{k+1}^{T} s_{k}\right]+\frac{d_{k}^{T} s_{k}}{d_{k}^{T} y_{k}}\left[t_{3} F_{k+1}^{T} y_{k}-t_{4} F_{k+1}^{T} s_{k}\right]\right)
$$

to obtain better results as in Section 5 .

\section{DERIVING THE PENALTY PARAMETER}

The derivation will be according to the two new parameters defined in (13) and (16), which we will be updated by achieving the condition of a sufficient descent direction for the CG-method as shown below:

\subsection{Lemma}

Assume that the generated method (13) with line search, then for a few positive scalars $\delta_{1}$ and $\delta_{2}$ satisfying $\delta_{1}+\delta_{2}<1$, we have:

$$
F_{k+1}^{T} d_{k+1} \leq-\left(1-\delta_{1}-\delta_{2}\right)\left\|F_{k+1}\right\|^{2}
$$

When

$$
\begin{aligned}
& \left|\xi_{k} t-1\right| \leq \sqrt{\frac{2 \delta_{2}\left(y_{k}^{T} s_{k}\right)}{\left\|s_{k}\right\|^{2}}} \\
& P_{1}=\frac{2 \delta_{1}\left\|F_{k+1}\right\|^{2}}{\left(t^{2}+1\right)\left\|\left(y_{k}-0.5 \lambda_{k} s_{k}\right)^{T} F_{k+1}\right\|^{2}} ; \quad \lambda_{k} \leq 1 \text { is a scalar. }
\end{aligned}
$$

Proof:

We have used (2) and (13) that: 


$$
\begin{gathered}
F_{k+1}^{T} d_{k+1}=-\left\|F_{k+1}\right\|^{2}+\frac{1}{\left(y_{k}^{T} s_{k}\right)^{2}}\left(\left(F_{k+1}^{T} y_{k}\right)\left(y_{k}^{T} s_{k}\right)\left(F_{k+1}^{T} s_{k}\right)-\frac{\xi_{k} t}{\left(t^{2}+1\right)}\left(F_{k+1}^{T} s_{k}\right)\left(y_{k}^{T} s_{k}\right)\left(F_{k+1}^{T} s_{k}\right)\right)- \\
\frac{\xi_{k}}{2 P_{1}\left(t^{2}+1\right)} \frac{\left(F_{k+1}^{T} s_{k}\right)^{2}}{\left(y_{k}^{T} s_{k}\right)^{2}}
\end{gathered}
$$

since $\lambda_{k} \leq 1$, implies that

$$
\begin{aligned}
F_{k+1}^{T} d_{k+1} \leq-\left\|F_{k+1}\right\|^{2} & +\frac{1}{\left(y_{k}^{T} s_{k}\right)^{2}}\left(\left(\left(y_{k}-0.5 \lambda_{k} s_{k}\right)^{T} F_{k+1}\right)\left(y_{k}^{T} s_{k}\right)\left(F_{k+1}^{T} s_{k}\right)\right) \\
& -\frac{\xi_{k}}{2 P_{1}\left(t^{2}+1\right)} \frac{\left(F_{k+1}^{T} s_{k}\right)^{2}}{\left(y_{k}^{T} s_{k}\right)^{2}}+\left(\frac{1}{2}-\frac{\xi_{k} t}{\left(t^{2}+1\right)}\right) \frac{1}{\left(y_{k}^{T} s_{k}\right)^{2}}\left(F_{k+1}^{T} s_{k}\right)\left(y_{k}^{T} s_{k}\right)\left(F_{k+1}^{T} s_{k}\right)
\end{aligned}
$$

Now, using the following inequality:

$$
x y \leq \frac{t^{\prime}}{4} x^{2}+\frac{1}{t^{\prime}} y^{2}
$$

Where $\mathrm{x}, \mathrm{y}$ and $t^{\prime}$ are positive scalars, we have:

$$
\begin{aligned}
F_{k+1}^{T} d_{k+1} & \leq-\left\|F_{k+1}\right\|^{2}+\frac{t^{\prime}}{4\left(y_{k}^{T} s_{k}\right)^{2}}\left(\left(y_{k}-0.5 \lambda_{k} s_{k}\right)^{T} F_{k+1}\right)^{2}\left(y_{k}^{T} s_{k}\right)^{2} \\
& +\frac{1}{\left(y_{k}^{T} s_{k}\right)^{2} t^{\prime}}\left(F_{k+1}^{T} s_{k}\right)^{2}-\frac{\xi_{k}}{2 P_{1}\left(y_{k}^{T} s_{k}\right)^{2}\left(t^{2}+1\right)}\left(F_{k+1}^{T} s_{k}\right)^{2}+\frac{\left(\xi_{k} t-1\right)^{2}}{2 y_{k}^{T} s_{k}\left(t^{2}+1\right)}\left(F_{k+1}^{T} s_{k}\right)^{2}
\end{aligned}
$$

Let $t^{\prime}=2 P_{1}\left(t^{2}+1\right)$,

$$
F_{k+1}^{T} d_{k+1} \leq-\left\|g_{k+1}\right\|^{2}+\frac{P_{1}\left(t^{2}+1\right)}{2}\left(\left(y_{k}-0.5 \lambda_{k} s_{k}\right)^{T} F_{k+1}\right)^{2}+\frac{\left(\xi_{k} t-1\right)^{2}}{2 y_{k}^{T} s_{k}\left(t^{2}+1\right)}\left(F_{k+1}^{T} s_{k}\right)^{2}
$$

By Cauchy-Schwarz inequality implies:

$$
\begin{gathered}
F_{k+1}^{T} d_{k+1} \leq-\left[1-\frac{P_{1}\left(t^{2}+1\right)}{2\left\|F_{k+1}\right\|^{2}}\left(\left(y_{k}-0.5 \lambda_{k} s_{k}\right)^{T} F_{k+1}\right)^{2}-\frac{\left(\xi_{k} t-1\right)^{2}}{2 y_{k}^{T} s_{k}\left(t^{2}+1\right)}\left\|s_{k}\right\|^{2}\right]\left\|F_{k+1}\right\|^{2} \\
F_{k+1}^{T} d_{k+1} \leq-\left(1-\delta_{1}-\delta_{2}\right) \leq-\rho_{1}\left\|F_{k+1}\right\|^{2}
\end{gathered}
$$

i.e.

$$
P_{1}=\frac{2 \delta_{1}\left\|F_{k+1}\right\|^{2}}{\left(t^{2}+1\right)\left\|\left(y_{k}-0.5 \lambda_{k} s_{k}\right)^{T} F_{k+1}\right\|^{2}} \quad \text { when } \quad\left|\xi_{k} t-1\right| \leq \sqrt{\frac{2 \delta_{2}\left(y_{k}^{T} s_{k}\right)}{\left\|s_{k}\right\|^{2}}}
$$

Since $t$ is an approximation of the step size, we use the following updated formula:

$$
t=\left\{\begin{array}{lr}
\xi_{k} & \text { if }\left|\xi_{k} t-1\right| \leq \sqrt{\frac{2 \delta_{2}\left(y_{k}^{T} s_{k}\right)}{\left\|s_{k}\right\|^{2}}} \\
1+\sqrt{\frac{2 \delta_{2}\left(y_{k}^{T} s_{k}\right)}{\left\|s_{k}\right\|^{2}}} & \text { O.W. }
\end{array}\right.
$$

Now the proof is completed.

\subsection{Lemma}

Assume that the newly generated method (16) with line search, then for a few positive scalars $\delta_{3}, \delta_{4}, \delta_{5}$ and $\delta_{6}$ satisfying $\delta_{3}+\delta_{4}+\delta_{5}+\delta_{6}<1$, we have:

$$
F_{k+1}^{T} d_{k+1} \leq-\left(1-\delta_{3}-\delta_{4}-\delta_{5}-\delta_{6}\right)\left\|F_{k+1}\right\|^{2}
$$

When 


$$
\begin{aligned}
& \left|t_{2}-2\right| \leq \sqrt{\frac{2 \delta_{4} \xi_{k}^{2}\left(y_{k}^{T} s_{k}\right)}{\left\|s_{k}\right\|^{2}}} \quad \& \quad\left|t_{4}-2\right| \leq \sqrt{2 \delta_{6} t^{2}\left(1-\tau_{k}\right)^{2}} \\
& \text { and } P_{2 a}=\frac{2 \delta_{3}\left\|F_{k+1}\right\|^{2}}{\left\|\left(t_{1} y_{k}-0.5 \lambda_{k} s_{k}\right)^{T} F_{k+1}\right\|^{2}} \quad \& \quad P_{2 b}=\frac{2 \delta_{5}\left\|F_{k+1}\right\|^{2}}{\left\|\left(t_{3} y_{k}-0.5 \lambda_{k} s_{k}\right)^{T} F_{k+1}\right\|^{2}}
\end{aligned}
$$

$\lambda_{k} \leq 1$ is a scalar.

Proof:

We substituting (16) in (6) and multiplying by $F_{k+1}$ that:

$$
\begin{aligned}
F_{k+1}^{T} d_{k+1}=-\| & F_{k+1} \|^{2} \\
& +\frac{1}{\varphi^{d}}\left(\left[t_{1}\left(F_{k+1}^{T} y_{k}\right)\left(y_{k}^{T} s_{k}\right)\left(F_{k+1}^{T} s_{k}\right)-t_{2}\left(F_{k+1}^{T} s_{k}\right)\left(y_{k}^{T} s_{k}\right)\left(F_{k+1}^{T} s_{k}\right)\right]\right. \\
& \left.+\frac{d_{k}^{T} s_{k}}{y_{k}^{T} s_{k}}\left[t_{3}\left(F y_{k}\right)\left(y_{k}^{T} s_{k}\right)\left(F_{k+1}^{T} s_{k}\right)-t_{4}\left(F_{k+1}^{T} s_{k}\right)\left(y_{k}^{T} s_{k}\right)\left(F_{k+1}^{T} s_{k}\right)\right]\right)-\frac{\xi_{k}}{2 P_{2} \varphi^{d}}\left(F_{k+1}^{T} s_{k}\right)^{2} \\
F_{k+1}^{T} d_{k+1}=-\left\|F_{k+1}\right\|^{2}+\frac{1}{\varphi^{d}}\left(\left(t_{1} y_{k}-0.5 \lambda_{k} s_{k}\right)^{T} F_{k+1}\right)\left(y_{k}^{T} s_{k}\right)\left(F_{k+1}^{T} s_{k}\right) & \\
+ & \frac{1}{\varphi^{d}}\left[\frac{1}{2}-t_{2}\right]\left(F_{k+1}^{T} s_{k}\right)\left(y_{k}^{T} s_{k}\right)\left(F_{k+1}^{T} s_{k}\right)+\frac{1}{\varphi^{d}}\left(\left(t_{3} y_{k}-0.5 \lambda_{k} s_{k}\right)^{T} F_{k+1}\right)\left(s_{k}^{T} s_{k}\right)\left(F_{k+1}^{T} s_{k}\right) \\
+ & \frac{1}{\varphi^{d}}\left[\frac{1}{2}-t_{4}\right]\left(F_{k+1}^{T} s_{k}\right)\left(s_{k}^{T} s_{k}\right)\left(F_{k+1}^{T} s_{k}\right)-\frac{\xi_{k}}{2 P_{2} \varphi^{d}}\left(F_{k+1}^{T} s_{k}\right)^{2}
\end{aligned}
$$

By following the same steps in Lemma 3.1 we get:

$$
\begin{aligned}
F_{k+1}^{T} d_{k+1} \leq-\| & F_{k+1} \|^{2}+P_{2 a}\left(\left(t_{1} y_{k}-0.5 \lambda_{k} s_{k}\right)^{T} F_{k+1}\right)^{2}+\frac{\left(t_{2}-2\right)^{2}}{2 \xi_{k}^{2} y_{k}^{T} s_{k}}\left(F_{k+1}^{T} s_{k}\right)^{2} \\
+ & P_{2 b}\left(\left(t_{3} y_{k}-0.5 \lambda_{k} s_{k}\right)^{T} F_{k+1}\right)^{2}+\frac{\left(t_{4}-2\right)^{2}}{2 t^{2}\left(1-\tau_{k}\right)^{2} s_{k}^{T} s_{k}}\left(F_{k+1}^{T} s_{k}\right)^{2}
\end{aligned}
$$

By Cauchy-Schwarz inequality implies:

$$
\begin{gathered}
F_{k+1}^{T} d_{k+1} \leq-\left[1-P_{2 a}\left\|t_{1} y_{k}-0.5 \lambda_{k} s_{k}\right\|^{2}-\frac{\left(t_{2}-2\right)^{2}}{2 \xi_{k}^{2} y_{k}^{T} s_{k}}\left\|s_{k}\right\|^{2}-P_{2 b}\left\|t_{3} y_{k}-0.5 \lambda_{k} s_{k}\right\|^{2}\right. \\
\left.-\frac{\left(t_{4}-2\right)^{2}}{2 t^{2}\left(1-\tau_{k}\right)^{2} s_{k}^{T} s_{k}}\left\|s_{k}\right\|^{2}\right]\left\|F_{k+1}\right\|^{2}
\end{gathered}
$$

Then,

$$
F_{k+1}^{T} d_{k+1} \leq-\left(1-\delta_{3}-\delta_{4}-\delta_{5}-\delta_{6}\right)\left\|F_{k+1}\right\|^{2} \leq-\rho_{2}\left\|F_{k+1}\right\|^{2}
$$

The proof is completed.

\subsection{Algorithm (PDL-CG) [29]}

Given $x_{0} \in \Omega, \gamma>0, r, \sigma, \varepsilon \in(0,1)$, stop test $\epsilon>0$, set $k=0$.

Step1: Evaluate $F\left(x_{k}\right)$ and test if $\left\|F\left(x_{k}\right)\right\| \leq \epsilon$ stop, else go to Step 3.

Step2: Generate the search direction $d_{k}$ by (6) and here $\beta_{k}^{P D L}=\frac{F_{k+1}^{T} y_{k}}{w_{k}^{T} s_{k}}-2\left\|y_{k}\right\|^{2} \frac{F_{k+1}^{T} s_{k}}{w_{k}^{T} s_{k}} ; w_{k}=y_{k}+$ $t_{k} d_{k}$ and $t_{k}=1+\max \left(0,-\frac{y_{k}^{T} d_{k}}{d_{k}^{T} d_{k}}\right)$. Stop if $d_{k}=0$.

Step3: Set $z_{k}=x_{k}+\alpha_{k} d_{k}$, where the step-size $\alpha_{k}=\max \left(\gamma r^{m} \mid m=0,1,2, ..\right)$ is determined by the line search $-F\left(x_{k}+\alpha_{k} d_{k}\right)^{T} d_{k}>\sigma \alpha_{k}\left\|d_{k}\right\|^{2}$

Step4: If $z_{k} \in \Omega$ and $\left\|F\left(z_{k}\right)\right\| \leq \epsilon$ stop, else compute the next point $x_{k+1}$ from Step (2).

Step5: Let $k=k+1$ and go to Step 1 . 


\subsection{New Algorithm (NDDL-CG).}

Step1: Given $x_{0} \in \Omega, \mathrm{r}, \eta, \sigma, \varepsilon \in(0,1)$, stop test $\epsilon>0$, set $k=0$.

Step2: Evaluate $F\left(x_{k}\right)$ and test if $\left\|F\left(x_{k}\right)\right\| \leq \epsilon$ stop, else go to Step 3.

Step3: When $y_{k}^{T} s_{k} \geq s_{k}^{T} s_{k}$ compute $P_{1}$ from (19) and if $P_{1} \neq \infty$ then $\beta_{k}^{\text {new1 } 1}$ from (13a) else from (13b).

Step4: When $y_{k}^{T} s_{k}<s_{k}^{T} s_{k}$ compute $P_{2}$ from (25) and if $P_{2} \neq \infty$ then $\beta_{k}^{\text {new2 }}$ from (16a) else from (16b)

Step5: Compute $d_{k}$ by (6) and stop if $d_{k}=0$.

Step6: Set $z_{k}=x_{k}+\alpha_{k} d_{k}$, where $\alpha_{k}=r^{m}$ with $\mathrm{m}$ being the smallest positive integer $\mathrm{m}$ such that:

$$
-F\left(x_{k}+\frac{r^{m}}{\varepsilon} d_{k}\right)^{T} d_{k}>\sigma \frac{r^{m}}{\varepsilon}\left\|d_{k}\right\|^{2}
$$

Step7: If $z_{k} \in \Omega$ and $\left\|F\left(z_{k}\right)\right\| \leq \epsilon$ stop, else compute the next point $x_{k+1}$ from (2).

Step8: Let $k=k+1$ and go to Step1.

\section{GLOBAL CONVERGENCE}

In the previous section, we gave a preface to the proof of convergence condition by establishing the property of sufficient descent through Lemmas 3.1 and 3.2. Now we need some assumption, to begin with, the proof of convergence condition, which is illustrated thus:

\subsection{Assumption}

Suppose $F$ fulfills the following assumptions:

a) The solution group of (2) is non-empty.

b) The function $F$ is Lipschitz continuous, i.e., there exists a positive constant $\mathrm{L}$ such that:

$$
\|F(x)-F(y)\| \leq L\|x-y\|, \forall x, y \in \mathbb{R}^{n}
$$

c) $F$ is uniformly monotone, that is,

$$
\langle F(x)-F(y), x-y\rangle \geq c\|x-y\|^{2}, \forall x, y \in \mathbb{R}^{n}, c>0
$$

\subsection{Lemma $[13]$}

assume $\left(\bar{x} \in \mathbb{R}^{n}\right)$ satisfy $F(\bar{x})=0$ and $\{x\}$ is generated by the new algorithm (NDDL-CG) that check Lemmas 3.1 and 3.2, then $\left\|x_{k+1}-\bar{x}\right\|^{2} \leq\left\|x_{k}-\bar{x}\right\|^{2}-\left\|x_{k+1}-x_{k}\right\|^{2}$. Specifically, it is $\{x\}$ bounded and

$$
\sum_{k=0}^{\infty}\left\|x_{k+1}-x_{k}\right\|<\infty
$$

\subsection{Lemma}

Suppose $\{x\}$ is generated by the new algorithm (NDDL-CG) then

$$
\lim _{k \rightarrow \infty} \alpha_{k}\left\|d_{k}\right\|=0
$$

\section{Proof:}

The sequence $\left\{\left\|x_{k}-\bar{x}\right\|\right\}$ not increasing, converging, and thus constrained. As well, $\left\{x_{k}\right\}$ is bounded and $\lim _{k \rightarrow \infty}\left\|x_{k+1}-x_{k}\right\|=0$. From (2) and used a line search, we have:

$$
\left\|x_{k+1}-x_{k}\right\|=\frac{\left|F\left(z_{k}\right)^{T}\left(x-z_{k}\right)\right|}{\left\|F\left(z_{k}\right)\right\|^{2}}\left\|F\left(z_{k}\right)\right\|=\frac{\left|\alpha_{k} F\left(z_{k}\right)^{T} d_{k}\right|}{\left\|F\left(z_{k}\right)\right\|} \geq \alpha_{k}\left\|d_{k}\right\| \geq 0
$$

Then the proof is completed.

\subsection{Theorem.}

Let $\left\{x_{k}\right\}$ and $\left\{z_{k}\right\}$ be the sequences generated by the new algorithm (NDDL-CG) then

$$
\liminf _{k \rightarrow \infty}\left\|F\left(x_{k}\right)\right\|=0 .
$$

Proof:

Case I: If $\lim \inf \left\|d_{k}\right\|_{k \rightarrow \infty}=0$, we have $\liminf _{k \rightarrow \infty}\left\|F\left(x_{k}\right)\right\|=0$. We use the continuity of $F$, the sequence $\left\{x_{k}\right\}$ has some accumulation point $\bar{x}$ such that $F(\bar{x})=0$. Since $\left\{\left\|x_{k}-\bar{x}\right\|\right\}$ converges and $\bar{x}$ is an accumulation point of $\left\{x_{k}\right\}$, it follows that converges to $\bar{x}$.

Case II: If lim inf $\left\|d_{k}\right\|_{k \rightarrow \infty}>0$, we have lim inf ${ }_{k \rightarrow \infty}\left\|F\left(x_{k}\right)\right\|>0$. By (29), it holds that $\lim _{k \rightarrow \infty} \alpha_{k}=0$. Using the line search: 


$$
-F\left(x_{k}+\frac{r^{m}}{\varepsilon} d_{k}\right)^{T} d_{k}<\sigma \frac{r^{m}}{\varepsilon}\left\|d_{k}\right\|^{2}
$$

and the boundedness of $\left\{x_{k}\right\},\left\{d_{k}\right\}$, we can choose a subsequence such that allowing $\mathrm{k}$ to go to infinity in the above inequality results

$$
-F(\bar{x})^{T} \breve{d} \leq 0
$$

On the other hand, from (17) and (23) we get

$$
-F(\bar{x})^{T} \check{d} \geq \rho_{i}\|F(\bar{x})\|^{2}>0
$$

For $\mathrm{i}=1$ and 2. It is through (31) and (32) indicates a contradiction. So, it is $\lim \inf { }_{k \rightarrow \infty}\left\|F\left(x_{k}\right)\right\|>0$ does not hold and the proof is complete.

\section{RESULTS AND EXPLANATIONS}

In this section, we present several results that explain the importance of the new algorithm (NDDLCG) compared to the standard Dai-Liao (PDL-CG) algorithm [20] using Matlab R2018b program in a laptop calculator with its Core ${ }^{\mathrm{TM}_{1}}$ specifications. The program finds the results on several non-derivative functions through several primary points indicated asshown in Table 1.

Table 1. Number of initial points

\begin{tabular}{cc}
\hline Name of Variable & Number \\
\hline$x_{1}$ & $(1,1,1, \ldots, 1)^{T}$ \\
$x_{2}$ & $(10,10,10, \ldots, 10)^{T}$ \\
$x_{3}$ & $\left(\frac{1}{2}, \frac{1}{2}, \frac{1}{2}, \ldots, \frac{1}{2}\right)^{T}$ \\
$x_{4}$ & $\left(\frac{1}{\mathrm{n}}, \frac{1}{\mathrm{n}}, \frac{1}{\mathrm{n}}, \ldots, \frac{1}{\mathrm{n}}\right)^{T}$ \\
$x_{5}$ & $\left(\frac{1}{10}, \frac{1}{10}, \frac{1}{10}, \ldots, \frac{1}{10}\right)^{T}$ \\
\hline
\end{tabular}

These algorithms are implemented for dimensions $\mathrm{n}(1000,2000,5000,7000,12000)$. The stopping scale is $\left\|F\left(x_{k}\right)\right\|<10^{-8}$. These algorithms are distinguished by their performance in (Iter): number of iterations, (Eval-F): number of function evaluations, (Time): CPU time in second and (Norm): the norm of approximation solution. The test problems are $F(x)=\left(f_{1}, f_{2}, f_{3}, \ldots, f_{n}\right)^{T}$ where $x=\left(x_{1}, x_{2}, x_{3}, \ldots, x_{n}\right)^{T}$, for $i=1,2, \ldots, n$ and $\Omega=\mathbb{R}_{+}^{n}$ Information of test functions as shown in Table 2.

Winner w.r.t. number of iterations, FVAL, TIME and NORM as shown in Table 3, according to all the initial points that we chose, shows the number of times the new algorithm has succeeded (NDDL-CG) versus each other against (PDL-CG) by relying on the number of iterations and on the number of times the goal function is calculated and on the time taken for each implementation In addition to the base value. As for Table 4, it represents the total implementation results of the (new (NDDL-CG) and old (PDL-CG)) algorithms) for each starting point of the initial five points.

Table 2. Information of test functions [31-36]

\begin{tabular}{ccc}
\hline Name of Functions & Details & Reference \\
\hline$F_{1}$ & $F_{i}(x)=2 x_{i}-\sin \left|x_{i}\right|$ & {$[31]$} \\
$F_{2}$ & $F_{i}(x)=x_{i}-\sin \left(x_{i}\right)$ & {$[31]$} \\
$F_{3}$ & $F_{i}(x)=e^{x_{i}}-1$ & {$[32]$} \\
$F_{4}$ & $F_{i}(x)=\ln \left(\left|x_{i}\right|+1\right)-\frac{x_{i}}{n}$ & {$[33]$} \\
$F_{5}$ & $F_{i}(x)=\min \left(\min \left(\left|x_{i}\right|, x_{i}^{2}\right), \max \left(\left|x_{i}\right|, x_{i}^{3}\right)\right)$ & {$[34]$} \\
$F_{6}$ & $F_{1}(x)=x_{1}-e^{\frac{\cos \left(x_{1}+x_{2}\right)}{n+1}}$ & {$[34]$} \\
& $F_{i}(x)=x_{i}-e^{\frac{\cos \left(x_{i+1}+x_{i}+x_{i-1}\right)}{n+1}}$, for $i=2,3, \ldots, n-1$ \\
& $F_{n}(x)=x_{n}-e^{\frac{\cos \left(x_{n-1}+x_{n}\right)}{n+1}}$ & \\
$F_{7}$ & $F_{i}(x)=\frac{i}{n} e^{x_{i}}-1$ & \\
\hline
\end{tabular}




\begin{tabular}{|c|c|c|}
\hline Name of Functions & Details & Reference \\
\hline$F_{8}$ & $F_{1}(x)=e^{x_{1}}-1, F_{i}(x)=e^{x_{i}}-x_{i-1}-1$ & [33] \\
\hline$F_{9}$ & $F_{i}(x)=\sum_{i=1}^{n}\left|x_{i}\right|^{i}$ & [35] \\
\hline$F_{10}$ & $F_{i}(x)=\sum^{n}\left|x_{i}\right| e^{-\sum_{i=1}^{n} \sin \left(x_{i}^{2}\right)}$ & [35] \\
\hline$F_{11}$ & $F_{i}(x)=\sum^{n}\left|x_{i} \sin \left(x_{i}\right)+0.1\left(x_{i}\right)\right|$ & [36] \\
\hline$F_{12}$ & $F_{i}(x)=\sum_{i=1}^{n}\left|x_{i}\right|^{i+1}$ & [36] \\
\hline
\end{tabular}

Table3. Winner w.r.t. number of iterations, FVAL, TIME and NORM

\begin{tabular}{ccc}
\hline Name of Variable & PDL-CG & NDDL-CG \\
& ITER / FVAL / TIME / NORM & ITER / FVAL / TIME / NORM \\
\hline$x_{1}$ & $35 / 35 / 24 / 26$ & $25 / 25 / 36 / 34$ \\
$x_{2}$ & $21 / 21 / 19 / 25$ & $39 / 39 / 41 / 35$ \\
$x_{3}$ & $15 / 15 / 11 / 18$ & $25 / 25 / 37 / 22$ \\
$x_{4}$ & $15 / 15 / 11 / 16$ & $10 / 10 / 14 / 9$ \\
$x_{5}$ & $15 / 11 / 3 / 20$ & $25 / 29 / 37 / 20$ \\
Total & $101 / 97 / 68 / 105$ & $124 / 128 / 165 / 120$ \\
\hline
\end{tabular}

Table4. Total of functions for each initial points

\begin{tabular}{ccc}
\hline Name of Variable & PDL-CG & NDDL-CG \\
& ITER / FVAL / TIME / NORM & ITER / FVAL / TIME / NORM \\
\hline$x_{1}$ & $1658 / 3612 / 1431.865 / 2.96 \mathrm{E}-07$ & $431 / 1136 / 931.698 / 1.26 \mathrm{E}-07$ \\
$x_{2}$ & $1758 / 3611 / 2441.866 / 2.96 \mathrm{E}-07$ & $382 / 990 / 835.6486 / 1.11 \mathrm{E}-07$ \\
$x_{3}$ & $1201 / 2607 / 1583.853 / 1.52 \mathrm{E}-07$ & $307 / 945 / 681.346 / 9.7 \mathrm{E}-08$ \\
$x_{4}$ & $135 / 680 / 1196.513 / 2.63 \mathrm{E}-08$ & $74 / 173 / 347.9768 / 1.65 \mathrm{E}-08$ \\
$x_{5}$ & $1678 / 4987 / 2678.288 / 1.89 \mathrm{E}-07$ & $226 / 652 / 458.5485 / 7.13 \mathrm{E}-08$ \\
Total & $6430 / 15497 / 9332.385 / 9.59 \mathrm{E}-07$ & $1420 / 3896 / 3255.2179 / 4.22 \mathrm{E}-07$ \\
\hline
\end{tabular}

Using Dolan and Mor'e [37] style, the following three figures are also for comparison between the two algorithms concerning the number of iterations, the number of times the function is calculated and the time is taken, which we calculated for the point $x_{5}$, Figure 1 shows the effect of the number of iterations on the two algorithms when switching and increasing dimensions. As for Figure 2, it is clear that the new algorithm is based on calculating the number of times the target function is better. Figure 3 shows the amount of time spent on the algorithms used in this work. As a conclusion, the figure shows us that the new algorithm is more efficient when compared to the old algorithm.

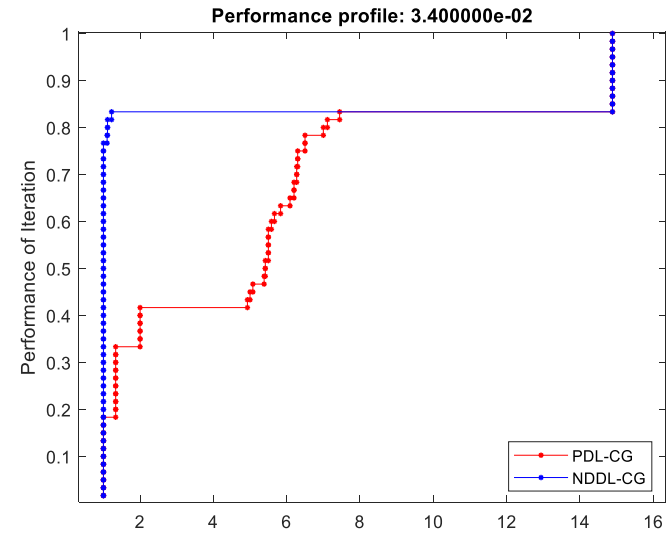

Figure 1. Performance of the two algorithms w.r.t. (Iter)

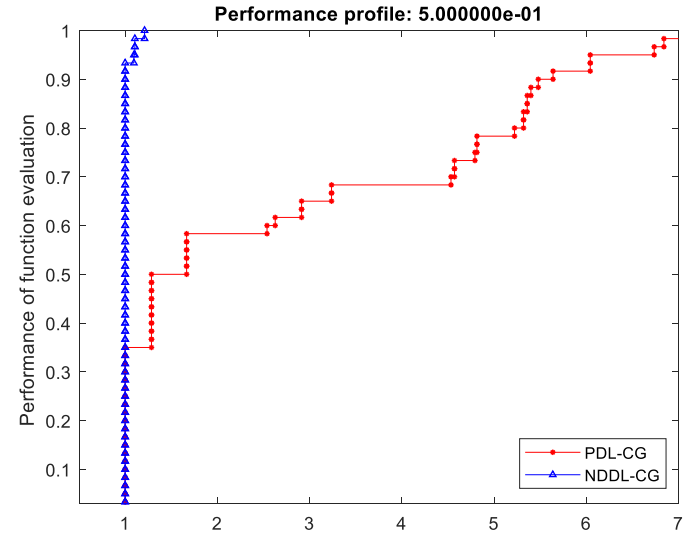

Figure 2. Performance of the two algorithms w.r.t. (Eval-F) 


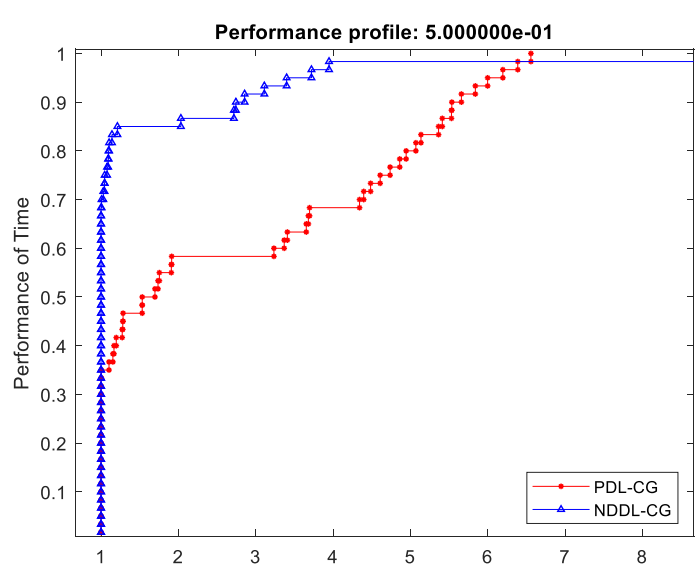

Figure 3. Performing the two algorithms w.r.t. (TIME)

\section{CONCLUSION}

From the results we conclude that the new algorithm (NDDL-CG) is more efficient than the old algorithm (PDL-CG) using most of the initial values when comparing its performance in changing dimensions. We also notice through the three drawings presented in the evaluation of previous results that the efficiency of the new algorithm increases with the increase in the number of dimensions and stability appears in some of the relevant results by calculating the goal function, and therefore the addition to the new algorithm (which contains the parameter of the penalty function) makes the new algorithm more appropriate than the algorithms Others are in the same field of work.

\section{Acknowledgments}

The research is supported by the College of Computer Sciences and Mathematics, University of Mosul, and College of Basic Education, Telafer University, Republic of Iraq. The authors declare that there are no conflicts of interest regarding this work.

\section{REFERENCES}

[1] J. Barzilai and J. M. Borwein, "Two-point step size gradient methods," IMA Journal of numerical analysis, vol. 8, no. 1, pp. 141-148, 1988.

[2] J. E. Dennis Jr and R. B. Schnabel, "Numerical methods for unconstrained optimization and nonlinear equations," SIAM, vol. 16, 1996.

[3] R. Z. Al-Kawaz, "Average Conjugate Gradient Method With Optimum Restart Powell For Nonlinear Function," International Journal of Computer Science and Information Security (IJCSIS), vol. 16, no. 8, 2018.

[4] A. Y. AL-Bayati and R. Z. AL-Kawaz, "Two New Extended PR Conjugate Gradient Methods for Solving Nonlinear Minimization Problems," Journal of Education and Science, vol. 27, no. 3, pp. 71-96, 2018.

[5] E. T. Hamed, H. I. Ahmed, and A. Y. Al-Bayati, "A New Hybrid Algorithm for Convex Nonlinear Unconstrained Optimization," Journal of Applied Mathematics, 2019.

[6] H. I. Ahmed, R. Z. Al-Kawaz and A. Y. Al-Bayati, "Spectral Three-Term Constrained Conjugate Gradient Algorithm for Function Minimizations," Journal of Applied Mathematics, 2019.

[7] E. T. Hamed, R. Z. Al-Kawaz and A. Y. Al-Bayati, "New Investigation for the Liu-Story Scaled Conjugate Gradient Method for Nonlinear Optimization," Journal of Mathematics, 2020.

[8] A. Y. Al-Bayati and M. M. M. Ali, "New multi-step three-term conjugate gradient algorithms with inexact line searches," Indonesian Journal of Electrical Engineering and Computer Science, vol. 19, no. 3, pp. 1564-1573, 2020.

[9] A. A. Al-Arbo1 and R. Z. Al-Kawaz, "Implementation of combined new optimal cuckoo algorithm with a gray wolf algorithm to solve unconstrained optimization nonlinear problems," Indonesian Journal of Electrical Engineering and Computer Science, vol. 19, no. 3, pp. 1582-1589, 2020.

[10] R. Z. Al-Kawaz, A. Y. Al-Bayati, M. S. Jameel, "Interaction between updated FR-CG algorithms with optimal Cuckoo algorithm," Indonesian Journal of Electrical Engineering and Computer Science, vol. 19, no. 3, 2020.

[11] R. Z. Al-Kawaz, H. I. Ahmed, and A. Y. Al-Bayati, "An efficient modified Cuckoo search optimization with the rational barrier function.", International Journal of Mathematics and Computer Applications Research (IJMCAR), vol. 8, no. 5, pp. 13-24, 2018.

[12] J. M. Ortega and W. C. Rheinboldt, "Iterative solution of nonlinear equations in several variables," SIAM, vol. 30, 1970. 
[13] M. V. Solodov and B. F. Svaiter, "A globally convergent inexact Newton method for systems of monotone equations," In Reformulation: Nonsmooth, Piecewise Smooth, Semismooth, and Smoothing Methods, Springer, pp. 355-369, 1998.

[14] Y. H. Dai, M. Al-Baali, and X. Yang, "A positive Barzilai-Borwein-like stepsize and an extension for symmetric linear systems," Numerical Analysis, and Optimization, Springer, Cham, pp. 59-75, 2015.

[15] C. Wang and Y. Wang, "A superlinearly convergent projection method for constrained systems of nonlinear equations," Journal of Global Optimization, vol. 44, no. 2, pp. 283-296, 2009.

[16] J. Liu and S. Li, "Spectral DY-type projection method for nonlinear monotone systems of equations," Journal of Computational Mathematics, vol. 33, no. 4, pp. 341-355, 2015.

[17] S. Y. Liu, Y. Y. Huang and H. W. Jiao, "Sufficient descent conjugate gradient methods for solving convex constrained nonlinear monotone equations," In Abstract and Applied Analysis, Hindawi, 2014.

[18] H. Mohammad and A. B. Abubakar, "A positive spectral gradient-like method for large-scale nonlinear monotone equations," Bulletin of Computational Applied Mathematics, vol. 5, no. 1, pp. 97-113, 2017.

[19] L. Zhang and W. Zhou, "Spectral gradient projection method for solving nonlinear monotone equations," Journal of Computational and Applied Mathematics, vol. 196, no. 2, pp. 478-484, 2006.

[20] C. Wang, Y. Wang and C. Xu, "A projection method for a system of nonlinear monotone equations with convex constraints," Mathematical Methods of Operations Research, vol. 66, no. 1, pp. 33-46, 2007.

[21] L. Zheng, "A new projection algorithm for solving a system of nonlinear equations with convex constraints," Bulletin of the Korean Mathematical Society, vol. 50, no. 3, pp. 823-832, 2013.

[22] Q. Li and D. H. Li, "A class of derivative-free methods for large-scale nonlinear monotone equations," IMA Journal of Numerical Analysis, vol. 31, no. 4, pp. 1625-1635, 2011.

[23] A. B. Abubakar, P. Kumam, and H. Mohammad, "A note on the spectral gradient projection method for nonlinear monotone equations with applications," Computational and Applied Mathematics, vol. 39, no. 129, pp. 1-35, 2020.

[24] M. Koorapetse and P. Kaelo, "Self-adaptive spectral conjugate gradient method for solving nonlinear monotone equations," Journal of the Egyptian Mathematical Society, vol. 28, no. 4, pp. 4, 2020.

[25] M. Ahookhosh, K. Amini and S. Bahrami, "Two derivative-free projection approaches for systems of large-scale nonlinear monotone equations," Numerical Algorithms, vol. 64, no. 1, pp. 21-42, 2013.

[26] Y. Ou and J. Li, "A new derivative-free SCG-type projection method for nonlinear monotone equations with convex constraints," J. Appl. Math. Comput, vol. 56, pp. 195-216, 2018.

[27] A. B. Abubakar, P. Kumam, H. Mohammad, A. M. Awwal and K. Sitthithakerngkiet, "A Modified FletcherReeves Conjugate Gradient Method for Monotone Nonlinear Equations with Some Applications," Mathematics, vol. 7, no. 8, pp. 745, 2019.

[28] Y. H. Dai and L. Z. Liao, "New conjugacy conditions, and related nonlinear conjugate gradient methods," Applied Mathematics and Optimization, vol. 43, no. 1, pp. 87-101, 2001.

[29] J. K. Liu and S. J. Li, "A projection method for convex constrained monotone non-linear equations with applications," Computers, and Mathematics with Applications, vol. 70, no. 10, pp. 2442-2453, 2015.

[30] M. Fatemi, "A new efficient conjugate gradient method for unconstrained optimization," Journal of Computational and Applied Mathematics, vol. 300, pp. 207-216, 2016.

[31] W. Zhou and D. Li, "Limited memory BFGS method for nonlinear monotone equations," Journal of Computational Mathematics, pp. 89-96, 2007.

[32] C. Wang, Y. Wang and C. Xu, "A projection method for a system of nonlinear monotone equations with convex constraints," Mathematical Methods of Operations Research, vol. 66, no. 1, pp. 33-46, 2007.

[33] W. La Cruz, J. Martínez and M. Raydan, "Spectral residual method without gradient information for solving largescale nonlinear systems of equations," Mathematics of Computation, vol. 75, no. 255, pp. 1429-1448, 2006.

[34] W. La Cruz, "A spectral algorithm for large-scale systems of nonlinear monotone equations," Numerical Algorithms, vol. 76, no. 4, pp. 1109-1130, 2017.

[35] X. S. Yang, "Test Problems in Optimization, Engineering Optimization: An Introduction with Metaheuristic Applications," John Wiley \& Sons, 2010.

[36] M. Jamil and X. S. Yang, "A literature survey of benchmark functions for global optimization problems," Int. Journal of Mathematical Modelling and Numerical Optimisation, vol. 4, no. 2, pp. 150-194, 2013.

[37] E. D. Dolan and J. J. Mor'e, "Benchmarking optimization software with performance profiles," Mathematical programming, vol. 91, no. 2, pp. 201-213, 2002. 